\title{
BMJ Open Lithium for Fracture Treatment (LiFT): a double-blind randomised control trial protocol
}

Diane Nam, ${ }^{1,2}$ Phumeena Balasuberamaniam (1) , ${ }^{1}$ Katrine Milner, ${ }^{1,3}$ Monica Kunz, ${ }^{1,3}$ Kathak Vachhani, ${ }^{4}$ Alex Kiss, ${ }^{5}$ Cari Whyne ${ }^{4,6}$
To cite: Nam D,

Balasuberamaniam P, Milner K, et al. Lithium for Fracture Treatment (LiFT): a doubleblind randomised control trial protocol. BMJ Open 2020;10:e031545. doi:10.1136/ bmjopen-2019-031545

- Prepublication history for this paper is available online. To view these files, please visit the journal online (http://dx.doi org/10.1136/bmjopen-2019031545).

Received 14 May 2019 Revised 10 December 2019 Accepted 16 December 2019
Check for updates

(C) Author(s) (or their employer(s)) 2020. Re-use permitted under CC BY-NC. No commercial re-use. See rights and permissions. Published by BMJ.

For numbered affiliations see end of article.

Correspondence to

Dr Diane Nam;

diane.nam@sunnybrook.ca

\section{ABSTRACT}

Introduction Fracture healing can fail in up to $10 \%$ of cases despite appropriate treatment. While lithium has been the standard treatment for bipolar disorder, it may also have a significant impact to increase bone healing in patients with long bone fractures. To translate this knowledge into clinical practice, a randomised clinical trial (RCT) is proposed.

Methods and analysis A multicentre double blind, placebo-controlled RCT is proposed to evaluate the efficacy of lithium to increase the rate and predictability of long bone fracture healing in healthy adults compared to lactose placebo treatment. 160 healthy individuals from 18 to 55 years of age presenting with shaft fractures of the femur, tibia/fibula, humerus or clavicle will be eligible. Fractures will be randomised to placebo (lactose) or treatment ( $300 \mathrm{mg}$ lithium carbonate) group within 2 weeks of the injury. The primary outcome measure will be radiographic union defined as visible callus bridging on three of the four cortices at the fracture site using a validated radiographic union score. Secondary outcome measures will include functional assessment and pain scoring.

Ethics and dissemination Participant confidentiality will be maintained with publication of results. Research Ethics Board Approval: Sunnybrook Research Institute (REB \# 356-2016). Health Canada Approval (HC6-24-C201560). Results of the main trial and secondary endpoints will be submitted for publication in a peer-reviewed journal and presented at conferences.

Trial registration number NCT02999022.

\section{INTRODUCTION}

Approximately, one-third of individuals will experience a fracture during their lifetime and in most cases, the reparative process results in fracture union after several weeks of immobilisation, surgery or both. Unfortunately, delayed or impaired bone healing can cause further disability which often necessitates extensive surgical interventions to promote union and restore normal skeletal function. ${ }^{1}$

While current Food and Drug Administration (FDA) approved anabolic drug treatments such as recombinant bone morphogenetic protein-2 (rhBMP-2), rhBMP-7 and

\section{Strengths and limitations of this study}

- This multicentre phase II randomised, double blind, placebo-controlled clinical trial will be the first to examine a $300 \mathrm{mg}$ dose of lithium carbonate in human fracture healing.

- The findings of this study will yield level 1 evidence to support or dismiss the efficacy of lithium to increase the rate and predictability of long bone fracture healing in healthy adults.

- This study will use validated measures such as the Radiographic Union Scoring to evaluate the primary outcome variable of fracture healing as well as the Visual Analogue Scale pain and RAND-36 functional assessments to evaluate the secondary outcomes.

- A key strength of this study is that patients will be stratified according to which long bone is fractured as well as whether they smoke. Due to the wellknown negative effects of smoking on bone healing, the findings of this study may provide strategies to improve fracture care for this particular group of patients.

- Our inclusion criteria limit the applicability of the trial results to healthy individuals with long bone fractures, thus further work will be needed to evaluate altered protocols suitable for individuals with osteoporotic or impaired bone healing conditions.

teriparatide (a recombinant fragment of the parathyroid hormone, PTH 1-34) are available, only rhBMP-2 is indicated for fracture management limited to open tibial shaft fractures. Despite positive evidence, these treatments have not gained consensus indication for use in fracture management due to limitations with respect to required local implantation, systemic injection and/or high costs. A simpler, economical, non-invasive approach to augment fracture repair that could decrease the overall healing duration and reduce the incidence of delayed union could dramatically improve health outcomes of fracture patients and alleviate the financial expenditures for healthcare systems worldwide. 
Lithium is an established psychiatric medication that has been used for the last 60 years in the treatment of bipolar disorder with demonstrated safe and effective administration over prolonged periods. A positive link has also been established between lithium and enhanced bone repair. Previous work has described lithium's association with increased bone turnover and bone formation by osteoblasts, as well as decreased bone resorption. ${ }^{23}$ One action of lithium is as a glycogen synthase kinase-3 3 (GSK-3 $\beta$ ) inhibitor that can activate the canonical Wingless (Wnt) $/ \beta$-catenin signalling pathway which is an important mechanism for increasing bone formation. ${ }^{4-6}$ The work of Chen $e t a l^{7}$ confirmed the positive effect of lithium administration on fracture repair and $\beta$-catenin activation via GSK-3 $\beta$ inhibition. In the early stages of fracture repair, $\beta$-catenin is needed to direct mesenchymal precursors into their respective chondrocyte and osteoblast lineages. Once mesenchymal precursors have committed to the osteoblast lineage, increasing $\beta$-catenin levels promote osteogenesis. ${ }^{3}$ Thus, to positively influence fracture-healing, stimulation of Wnt/ $\beta$-catenin signalling must occur after mesenchymal precursors have been committed to the osteoblast lineage. The ideal therapeutic influence of lithium must target the timing when mesenchymal precursors become committed to the osteoblast lineage, ${ }^{7}$ a cellular transition closely linked to the physiological shift from soft to hard callus during endochondral (secondary) fracture repair. While the exact timing is not conclusive, evidence suggests that this soft to-hard callus transition peaks between 7 and 15 days after fracture in rodents. ${ }^{8-10}$ Therefore, lithium therapy aimed at enhancing fracture-healing targets during this time range will be most effective.

Our previous published work determined the optimal oral administration parameters for lithium treatment $(20 \mathrm{mg} / \mathrm{kg}$, administered 7 days post fracture, for 2 weeks) in rodents which led to a $46 \%$ increase in torsional strength of healing femoral fractures at 4 weeks compared to untreated controls. ${ }^{11}$ No differences in biomechanical strength/bone density were seen between treated and untreated non-fractured contralateral limbs. Similar improvements in strength were seen in lithium treated fractures in osteoporotic animals but only when the start of treatment was delayed as the actions of lithium on the (Wnt) / $\beta$-catenin signalling pathway during the reparative phase in osteoporosis was determined as later. ${ }^{12}$ In order to translate the previously identified dosage parameters for the use of lithium for fracture healing in humans, we used the interspecies dose translation approach outlined by Reagen Shaw and others ${ }^{13}$ which was based on FDA guidelines. This approach is based on body surface area to better reflect basal metabolism rather than only body weight. Using this methodology, $20 \mathrm{mg} / \mathrm{kg}$ in a rat translated to $3.24 \mathrm{mg} / \mathrm{kg}$ in humans. From this calculation as well as the analysis of the pharmacokinetics of lithium and permissible serum lithium levels in humans, ${ }^{14}$ a dose of $300 \mathrm{mg} /$ day was selected for this trial. There have been no previous studies using this low dose in human fracture healing or psychiatric treatments.
This paper presents the rationale and protocol for a double blind, multicentre, randomised phase II clinical trial to evaluate the effect of an equivalent lithium treatment regimen ${ }^{13}$ in healthy patients presenting with longbone fractures. It is hypothesised that low dose lithium treatment (300 mg orally/day) commencing 2 weeks post fracture for a short 2-week duration will significantly increase long bone fracture healing compared to lactose placebo treatment.

\section{Study objectives}

The primary objective of this study is to determine if a low oral dose lithium treatment $(300 \mathrm{mg} /$ day $)$ commencing 2 weeks post-fracture (for non-operative patients) or surgery (for operative patients) for a duration of 14 days will significantly increase the rate and predictability of long bone fracture healing compared to lactose placebo treatment in healthy adults. The secondary objectives of this study are to determine if lithium treatment can improve patient pain and function compared to placebo.

\section{METHODS}

\section{Protocol design}

Lithium for Fracture Treatment (LiFT) is a multicentre randomised, double blind, placebo-controlled, superiority trial with two parallel groups and a primary endpoint of radiographic healing assessed at 4, 6, 8, 12 and 24 weeks post fracture or surgery. One hundred and sixty healthy individuals will be randomised $1: 1,300 \mathrm{mg}$ lithium carbonate versus lactose placebo, and a minimisation procedure will stratify participants based on which long bone is fractured and smoking status.

The groups to be randomised to either lithium or placebo will be:

1. Upper extremity (clavicle or humerus) fracture smoking.

2. Upper extremity (clavicle or humerus) fracture nonsmoking.

3. Lower extremity (femur or tibia/fibula) fracture smoking.

4. Lower extremity (femur or tibia/fibula) fracture non-smoking.

The study drug will be administered orally once daily (evening) for a total of 2 weeks starting 14 days post fracture (for non-operative patients) or surgery (for operative patients). Follow-up clinic visits $(30-60 \mathrm{~min})$ will occur at 4, 6, 8, 12 and 24 weeks post fracture/surgery. A serum lithium level will be sampled on day 7 of the study drug administration period. Ongoing clinical care by the treating surgeon will continue beyond 24 weeks if required. Follow-up data may be collected remotely (eg, by phone or email) for the following visits. An overview of the schedule of study events is outlined in table 1 .

\section{Sample size considerations}

The sample size calculation was based on pilot data analysis previously completed comparing mean Radiographic 
Union Scores (RUSTs) between treatment and placebo at 24 weeks in the pilot study. With the mean RUST of the placebo taken to be 8.25 and an SD of 2.6 based on our pilot data, a two-sample two-sided t-test with 70 per group has $80 \%$ power at alpha of 0.05 to test a difference in means of 1.24 or greater. Assuming a drop-out rate of $10 \%-15 \%$, this sample size will be inflated to 80 per group, or 160 participants in total. The participants will be randomised 1:1 into the two groups and stratified according to long bone and smoking. The sample size calculation was run using PASS V.12 (Hintze, J. (2014), NCSS; Kaysville, Utah, USA). A single interim analysis will be carried out at $50 \%$ recruitment. The O'Brien-Fleming function was used with the sample size estimates to partition the alpha for the interim and final analysis to approximately 0.003 and 0.047 , respectively.

\section{Randomisation}

Treatment allocation will be assigned using a specialised computerised randomisation system and dispensed by the Sunnybrook Drug Information Services which will ensure that concealment can never be breached. The unmasked primary site pharmacist will prepare bottles of either lithium carbonate or placebo according to the randomisation allocation. Participants will be randomised 1:1 into one of the two groups and stratified according to long bone location and smoking status using a minimisation algorithm (minimal sufficient balance, MSB). The MSB randomisation is a minimisation procedure that preserves balance in smaller trials, where imbalances in important baseline prognostic variables may occur by chance and confound the primary outcome. ${ }^{15}$ It preserves a greater degree of randomness in patient allocation compared to permuted block designs. ${ }^{15}$

\section{Blinding}

All participants/staff except the pharmacist preparing the drug will be blinded to the intervention groups. Blinding is achieved by overencapsulation of each treatment such that active and placebo medications will appear identical. Capsule filler powder consisting of microcrystalline cellulose: lactose 1:1 will be used. A 'no objection' letter will be obtained from the Therapeutic Products Directorate of Health, Canada, to use lithium carbonate for fracture treatment prior to the initiation of the study. Emergency unblinding will be allowed if participants present with an adverse event that in the opinion of consulting physicians is not explained by other causes and knowing the treatment allocation will aid in the patient's medical management.

\section{Eligibility criteria}

Individuals, who meet the inclusion and exclusion criteria outlined in table 2, will be considered for this trial.

\section{Participant screening and recruitment}

The study will be completed across five hospitals throughout Ontario. Figure 1 provides an overview of the trial procedure, including the patient identification and screening procedures. Participants who meet the eligibility criteria after preliminary screening will be approached by their surgeon or others in their circle of care to obtain permission for research staff to approach them. Research staff will then explain the nature of the trial and conduct 
Table 2 Inclusion and exclusion criteria

\section{Inclusion Criteria}

\section{1) Age between 18-55}

2) Diaphyseal fractures (OTA diaphyseal subclass $2 A$ or $B$ ) of the humerus, femur, tibia/fibula, or (OTA diaphyseal subclass B1 or B2) clavicle with or without nerve injury

\section{3) ASA class $\leq 2$ prior to injury \\ 4) Primarily closed or open fractures that have complete} wound coverage

\section{5) Fracture date $\leq 14$ days}

6) Fractures treated surgically or non-surgically by endochondral/secondary bone healing

7) Date of surgery $\leq 3$ days from fracture date, if fracture is treated surgically

\section{Exclusion Criteria}

\section{1) Renal impairment}

2) Currently taking lithium or anti-psychotic or anti-seizure medication for the treatment of these conditions, or at the discretion of an investigator

\section{3) Pregnant or breastfeeding}

4) Hypothyroidism that, in the opinion of an investigator, is not medically controlled

\section{5) Past allergy or adverse reaction to lithium}

6) Autoimmune disease that, in the opinion of an investigator, is not medically controlled

7) Malignancy that, in the opinion of an investigator, is not medically controlled

8) Lactose intolerance

9) Fractures surgically treated with absolute stability/primary bone healing

10) Tibia fractures with intact fibula

11) Metabolic bone disease that, in the opinion of an investigator, is not medically controlled

12) Inability to be compliant with study protocol in the opinion of investigator.

* Othopaedic Trauma Association.

${ }^{* *}$ American Society of Anesthesiologists.

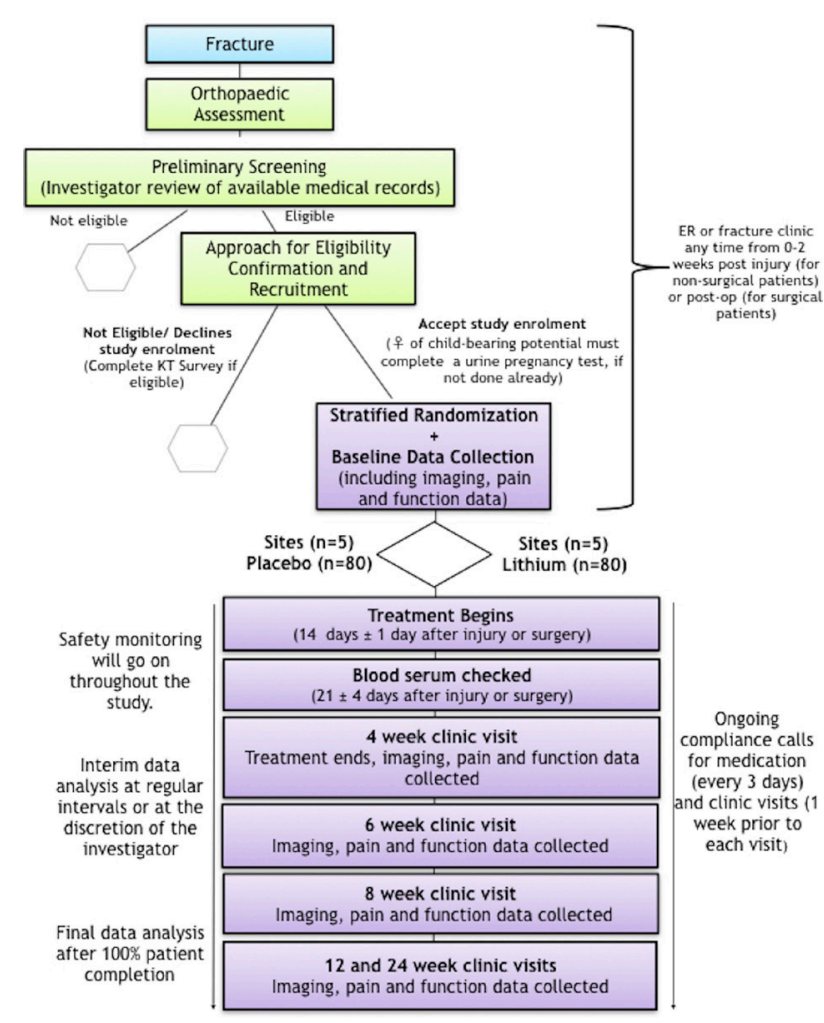

Figure 1 Overview of trial procedure. Clinic visit dates are relative to date of injury (for non-operative patients) or surgery (for operative patients). KT Survey = knowledge translation survey; ER = emergency department. an interview reviewing their medical history to confirm eligibility. Additional educational materials will be provided to patients to increase their knowledge about fracture healing, lithium and the proposed clinical use of lithium in fracture healing. Eligible participants will then be asked to sign an informed consent form. This can take place anytime within 14 days post fracture (for nonsurgical patients) or postop (for surgical patients).

Research staff will document reasons for failure to randomise any eligible patient and ask all eligible patients to complete a short survey (iPad or paper) which will specifically focus on patients' knowledge of lithium in the context of psychiatric illness and their willingness to consider this drug's use to improve fracture healing. This non-identifiable data will be held in a password-protected and secure cloud server in Canada. This information will be used to guide future knowledge translation efforts towards ensuring that current perceptions of lithium do not pose a barrier to its orthopaedic use.

\section{Concomitant care}

Surgery

The participant and surgeon will determine operative or non-operative treatment based on the injury characteristics and best clinical practice. Participation in Lithium for Fracture Treatment (LiFT) will not affect this management in anyway. To ensure that participants meet inclusion criteria 6 and 7 in table 2, randomisation for identified patients will only occur after surgery is completed. 


\section{Medications}

Routine analgesics may be used as required. Non-steroidal anti-inflammatory drugs (NSAIDs) are prostaglandin inhibitors. Currently, there is an absence of clear clinical or scientific evidence reporting that NSAIDs pose harm to normal bone healing. ${ }^{16}$ Thus, NSAIDs will be considered a risk factor and recorded in the study information for the final data analysis and not excluded.

\section{Smoking}

The negative effects of smoking on bone healing are well known in the literature. ${ }^{17}$ However, smokers are not excluded as the effect of lithium in this sub-population is of significant interest to the study. Smoker randomisation will be stratified to ensure a balance of patients receiving lithium and placebo.

\section{Rehabilitation/activity}

Specific activity instructions or weight-bearing restrictions are not required for the study.

\section{Study interventions}

Eligible participants who provide written consent will commence the study treatment of a low-dose lithium treatment $(300 \mathrm{mg} /$ day $)$ or placebo 14 days post injury or surgery. The short 2-week oral administration will facilitate adherence. Study staff will phone patients regularly during this period to ensure compliance and document adherence on the case report form (CRF). Women of childbearing potential must have a negative urine pregnancy test prior to randomisation. Adherence checks with pill counts will be completed and recorded at the 4-week follow-up visit.

Modifications to the allocated treatment will include discontinuing the medication for any renal abnormalities or symptoms of hypothyroidism and will be reported as adverse events. A serum lithium level will be sampled on day 7 of the study drug administration period.

\section{Study outcomes}

Primary outcome measure

The primary study endpoint is radiographic healing assessed at five time-points after injury (for non-surgical patients) or surgery (for surgical patients). Radiographic union is the clinical standard for evaluating fracture healing at routine clinic follow-ups. X-rays will be taken at presentation to hospital, 4, 6, 8, 12 and 24 weeks post fracture/surgery and assessed by the participant's surgeon to monitor progression of healing. Union will be defined as a visible callus bridging on three of four cortices at the fracture site in the anteroposterior and lateral radiographs using a previously validated RUST score. ${ }^{18} 19$ Table 3 provides an assessment tool that surgeons may use to collect this measure. While it is anticipated that lithium will be beneficial for all fractures healing by endochondral ossification, only diaphyseal fractures subtype $2 \mathrm{~A}$ and $2 \mathrm{~B}$ were included to facilitate ease of scoring to identify the fracture callus as extensively multifragmentary or segmental fractures will be challenging to score consistently.

\section{Secondary outcome measures}

Secondary outcome measures of pain and function are assessed at each follow-up visit. Pain scoring entails patient completion of a Visual Analogue Scale (VAS) to describe the pain across a continuum (none to extreme).$^{20}$ Functional assessment involves completion of the RAND-36, multipurpose, short-form health survey of psychometrically based physical and mental health summary measures. ${ }^{21}$ Case report forms documenting use of NSAIDs, (re)operation after initial fracture management if applicable, and other complications and unforeseen events are completed for all patients. The surgeon performs a clinical exam and notes any complications with the research coordinator in addition to providing routine follow-up care. These outcomes are assessed at $4,6,8,12$ and 24 weeks post fracture (for non-operative patients) or post-op (for operative patients).

\section{Trial monitoring}

Sunnybrook's Centre for Clinical Trials Support (CCTS) will monitor the trial including site initiation visits, site close-out visits and interim monitoring visits which may include in-person and remote activity.

A data safety monitoring board (DSMB) has been established, comprising an orthopaedic surgeon, a clinical trialist and a biostatistician experienced in RCTs but not involved with this clinical trial. All lithium serum level results $>1.2 \mathrm{mEq} / \mathrm{L}^{21}$ are reported by the laboratory directly to the DSMB for review. An interim analysis will be conducted at regular intervals as required or at the

Table 3 Assessment tool for Radiographic Union Score (RUST)

$\begin{array}{cllll}\text { Assessment Tool for Radiographic Union (RUST Score) } & & \\ \text { Fortex } & \text { Fracture Line, } & \text { Fracture Line, } & \text { No Fracture Line, } & \text { Total Score: } \\ & \text { No Callus } & \text { Visible Callus } & \text { Bridging Callus } & \text { Minimum = 4 } \\ & (\text { Score=1) } & \text { (Score=2) } & \text { (Score=3) } & \text { Maximum = 12 }\end{array}$

\section{Anterior}

Posterior

Lateral

Medial 
discretion of the principal investigator. Interim analysis results will be shared with the DSMB for discussion and recommendations. Amendments will require review and approval by each site's research ethics board (REB), the DSMB and Health Canada before the changes are implemented, unless to eliminate an immediate hazard. All outcome measures will be evaluated at the completion of the trial when the last participant has reached 24 weeks post-fracture.

\section{Study follow-up}

Table 1 shows the schedule of study events. Baseline data including age, sex/gender, body mass index, occupation, hand dominance, Workers Compensation and smoking status, and pain and function score are obtained by research staff, at the time of consent. Telephone contact with the participants is scheduled every 3 days during the 2-week drug treatment phase and 1 week prior to each subsequent clinic visit to answer questions and reinforce compliance. Missed follow-up visits or early withdrawal will be documented.

The study drug will be discontinued in participants who become pregnant. Because the upper limit of normal for serum lithium is $1.3 \mathrm{mmol} / \mathrm{L}$, the study drug will be discontinued in participants whose serum lithium level is at or above $1.1 \mathrm{mmol} / \mathrm{L}$; although we do not expect to see this result with the low dose being used in this study. These participants will remain in the study and be included in the intent-to-treat analysis.

Participants may stop taking the drug treatment and continue with further visits as per protocol or withdraw from the study at any time for any reason. Participants withdrawing from the study will be contacted by the study research team requesting a final visit, follow-up with any unresolved adverse events and document the reason for withdrawal. No further study procedures or evaluations will be performed without obtaining explicit permission. Study participants may also be withdrawn from the study at the discretion of an investigator for reasons such as, but not limited to safety, participant compliance or behavioural concerns. Any data collected prior to the withdrawal of consent will be retained and used.

\section{Data management}

Sunnybrook's Centre for Clinical Trial Support (CCTS) provides data acquisition, management and analytic coordination. Data will be collected through a secure password, web-based Electronic Data Capture (EDC) System that facilitates data entry, review and modification. Information is submitted through the EDC system via internet in encrypted form to the data storage system. Programming and validation of the electronic case report forms will take place at CCTS.

\section{Return and destruction of investigational product}

At the completion of the study, there will be a final reconciliation of investigational product shipped, used and remaining. This reconciliation will be logged, signed and dated. Any discrepancies noted will be investigated, resolved and documented prior to return or destruction of unused investigational product. Investigational product destroyed on site will be documented in the study files.

\section{Statistical methods}

Descriptive statistics will be calculated for all variables of interest. Continuous measures such as age will be summarised using means and standard deviation (SD); categorical measures will be summarised using counts and percentages. A 95\% confidence interval (CI) will be calculated for percentage of long bone healed for the overall sample as well as each strata: clavicle, humerus, femur and tibia/fibula. CI will also be presented for smokers and non-smokers. A two-sample, two-sided t-test (or Wilcoxon rank sum test should data be non-normally distributed) will be used to compare the two groups (treatment vs placebo) on their RUST (range 4-12). To assess those with scores $\geq 9$ on this measure, a $\chi^{2}$ test (or Fisher's exact test for the case of low expected counts) will be used to compare groups based on this binary variable. For the secondary measures, the VAS and RAND36 scales mean score will be compared between groups using a twosample t-test. All analyses will be carried out using SAS V.9.3 (SAS Institute).

\section{Missing data}

The study population analysis will be intention-to-treat. Missing data will be carried forward from the last intake for radiographic union, VAS and SF36 if RUST $\geq 9$, VAS $\geq 75$ and RAND36 $\geq 75$. The values indicated for these measures are close to normal and expected to remain stable unless a new event occurs. Later assessments will not be used to fill in earlier missing data.

\section{Ethical considerations}

All patients are provided an REB approved consent form describing the study and providing sufficient information to make an informed decision about their participation in this study. The formal consent of a patient, using the $\mathrm{REB} /$ (Institutional review board (IRB)-approved consent form, must be obtained before that patient undergoes any study procedure. Medical care will be provided for patients, if they become sick or injured as a direct result of their participation in this study.

\section{Safety stopping rules}

Study termination will be considered if any of the stopping criteria is satisfied, to ensure the safety of participants.

1. Withdrawal of lithium from the Canadian market by Health Canada. Withdrawal from other markets will be reviewed and a recommendation about the continuation of the study provided to the principal investigator (PI).

2. Occurrence of one unexpected event leading to the death that is definitely/probably due to the study drug (lithium).

3. Statistically significant increase in serious adverse events (SAEs) in lithium compared to placebo group 
4. that are definitely/probably related to lithium at the time of the interim analysis.

\section{Patient and public involvement}

Patients and members of the public were not invited to comment on the study design and were not consulted to develop patient relevant outcomes or interpret the results. Patients were not invited to contribute to the writing or editing of this document for readability or accuracy.

\section{DISCUSSION}

Fractures are frequent orthopaedic injuries afflicting millions of working adults. Accelerating fracture healing in otherwise healthy individuals would speed return to work and reduce the burden of injury. This paper presents rationale for, and organisation of, the LiFT study, detailing the protocol for a double blind, multicentre, randomised controlled clinical trial to evaluate the effect of a defined lithium treatment regimen in healthy patients presenting with long-bone fractures.

Lithium treatment represents a simple, targeted and clinically translatable pharmacological approach for managing patients with fractures to accelerate and/or predictably heal bone. The optimal timing of lithium's action in the cascade of fracture healing is uniquely advantageous for clinical applications since patients who sustain a fracture are referred to the attention of an orthopaedic surgeon typically in the first 2 weeks post injury. As such, lithium treatment eligibility is captured and perfectly timed during this period. The low dose lithium treatment regimen employed in this study also minimises the risk of potential side effects. Lithium side effects and toxicity are primarily acute and related to doses that yield serum levels above the recommended upper limit of $1.2 \mathrm{mEq} / \mathrm{L} .{ }^{13}$ Normally, clinical dosing for psychiatric indications begins at $300 \mathrm{mg} /$ day, increasing to a maintenance serum level of $0.8 \mathrm{mEq} / \mathrm{L}$ or $900-1200 \mathrm{mg} /$ day (less for the elderly and children) ${ }^{22}$ Thus, the lower dose of $300 \mathrm{mg}$ /day of lithium used in this trial is considered safe and will substantially reduce its potential adverse systemic effects as lithium toxicity is dose dependent. To the best of our knowledge, no previous studies of this low dosage are outlined in the literature. This suggests that the lithium levels needed to improve bone healing come with minimal risk of acute toxicity. While we do not anticipate any dose-dependent toxicity given the very low dosage and short duration of treatment, due to the narrow therapeutic-to-toxic ratio of lithium, a serum lithium level will be sampled 7 days post drug administration for study participants. While there are long-term side effect concerns, including hyperparathyroidism, hypothyroidism and nephrogenic diabetes insipidus, in patients managed with lithium, ${ }^{23-25}$ they are less relevant for the short-term use of lithium in this trial. The full list of the expected adverse events associated with lithium carbonate is available by its product monograph (https://pdf.hres. ca/dpd_pm/00024251.PDF).
In the elderly, osteoporotic fractures are increasing at an alarming rate and achieving union is critical to prevent their ensuing decline in health and autonomy. ${ }^{26} 27$ The knowledge gained from this study will set the foundation in designing future randomised clinical trials to apply lithium rescue in osteoporosis and other conditions of impaired bone healing such as infection, malignancy and metabolic bone disorders. In considering wider clinical adoption of lithium for fracture treatment, it is critical to understand potential barriers and current perceptions of lithium that may impede clinical uptake. In our pilot work, we conducted a series of surveys designed to understand perceptions of the general public, patients with long bone fractures and orthopaedic surgeons towards the use of lithium in the context of fracture treatment. ${ }^{28}$ In all three groups, we identified a lack of knowledge about lithium, combined with barriers specific to lithium's current use as a psychiatric drug. As such, concurrent with the LiFT trial described in this protocol we propose to develop comprehensive educational materials for clinicians and patients with long bone fractures that will be essential to guide future knowledge translation efforts towards ensuring that both lack of knowledge and current perceptions of lithium do not pose a barrier to its orthopaedic use.

The results of the LiFT trial will make an important contribution to orthopaedic literature and, if positive, are likely to lead to rapid changes in fracture management practice. Demonstrating a benefit towards accelerated and/or more reliable healing of long bone fractures with short term, low dose lithium administration has the potential to improve the lives of fracture patients worldwide and to reduce the economic burden associated with long bone fractures and impaired bone healing. The benefits of a widely accessible, safe, low-cost, low-dose and short-duration therapy with ease of administration gives tremendous promise to lithium's potential to significantly change global orthopaedic clinical practice.

\section{Author affiliations}

${ }^{1}$ Division of Orthopaedic Surgery, Sunnybrook Health Sciences Centre, Toronto, Ontario, Canada

${ }^{2}$ Division of Orthopaedic Surgery, University of Toronto, Toronto, Ontario, Canada ${ }^{3}$ Division of Orthopaedic Surgery, Holland Orthopaedic and Arthritic Centre, Toronto, Ontario, Canada

${ }^{4}$ Orthopaedic Biomechanics Lab, Sunnybrook Health Sciences Centre, Toronto, Ontario, Canada

${ }^{5}$ Research Design and Biostatistics, Sunnybrook Health Sciences Centre, Toronto, Ontario, Canada

${ }^{6}$ Department of Orthopaedic Surgery and Institute of Biomaterials \& Biomedical Engineering, University of Toronto, Toronto, Ontario, Canada

Contributors DN and CW, were involved in the concept development and funding applications. KM, MK and PB were involved in the ethics application, participant recruitment, data collection and study management. DN, PB, CW and KV participated in the manuscript preparation and editing/review. DN, KV and AK were involved in the study statistical design. All authors contributed to the final approval of this manuscript.

Funding This work was supported by ClHR (2016- HC6-24-C201560).

Competing interests None declared. 
Patient consent for publication Not required.

Ethics approval Centre for Clinical Trial Support, Sunnybrook Research Institute (356-2016) Health Canada (HC6-24-c201560).

Provenance and peer review Not commissioned; externally peer reviewed.

Data availability statement Data sharing not applicable as no datasets generated and/or analysed for this study.

Open access This is an open access article distributed in accordance with the Creative Commons Attribution Non Commercial (CC BY-NC 4.0) license, which permits others to distribute, remix, adapt, build upon this work non-commercially, and license their derivative works on different terms, provided the original work is properly cited, appropriate credit is given, any changes made indicated, and the use is non-commercial. See: http://creativecommons.org/licenses/by-nc/4.0/.

\section{ORCID iD}

Phumeena Balasuberamaniam http://orcid.org/0000-0003-4492-3352

\section{REFERENCES}

1 MacKenzie EJ, Bosse MJ, Kellam JF, et al. Early predictors of long-term work disability after major limb trauma. $J$ Trauma 2006;61:688-94.

2 Spencer GJet al. Wnt signalling in osteoblasts regulates expression of the receptor activator of NF B ligand and inhibits osteoclastogenesis in vitro. J Cell Sci 2006;119:1283-96.

3 Hill TP, Später D, Taketo MM, et al. Canonical Wnt/ $\beta$-catenin signaling prevents osteoblasts from differentiating into chondrocytes. Dev Cell 2005;8:727-38.

4 Hoeppner LH, Secreto FJ, Westendorf JJ. Wnt signaling as a therapeutic target for bone diseases. Expert Opin Ther Targets 2009;13:485-96.

5 Clément-Lacroix P, Ai M, Morvan F, et al. Lrp5-independent activation of Wnt signaling by lithium chloride increases bone formation and bone mass in mice. Proc Natl Acad Sci U S A 2005;102:17406-11.

6 Kulkarni NH, Onyia JE, Zeng Q, et al. Orally bioavailable GSK3alpha/beta dual inhibitor increases markers of cellular differentiation in vitro and bone mass in vivo. J Bone Miner Res 2006;21:910-20.

7 Chen Y, Whetstone HC, Lin AC, et al. Beta-Catenin signaling plays a disparate role in different phases of fracture repair: implications for therapy to improve bone healing. PLoS Med 2007;4:e249.

8 Strohbach CA, Strong DD, Rundle CH. Gene therapy applications for fracture repair. In: Kang C, ed. Gene therapy applications. Shanghai: Intech, 2011: 27.

9 Marsell R, Einhorn TA. The biology of fracture healing. Injury 2011;42:551-5.

10 Hadjiargyrou M, Lombardo F, Zhao S, et al. Transcriptional profiling of bone regeneration. insight into the molecular complexity of wound repair. J Biol Chem 2002;277:30177-82.
11 Bernick J, Wang Y, Sigal IA, et al. Parameters for lithium treatment are critical in its enhancement of fracture-healing in rodents. $J$ Bone Joint Surg Am 2014;96:1990-8.

12 Vachhani K, Whyne C, Wang Y, et al. Low-Dose lithium regimen enhances endochondral fracture healing in osteoporotic rodent bone. J Orthop Res 2018;36:1783-9.

13 Reagan-Shaw S, Nihal M, Ahmad N. Dose translation from animal to human studies revisited. Faseb J 2008;22:659-61.

14 Guelen PJM, Janssen TJ, de Witte TCM, et al. Bioavailability of lithium from lithium citrate syrup versus conventional lithium carbonate tablets. Biopharm Drug Dispos 1992;13:503-11.

15 Zhao W, Hill MD, Palesch Y. Minimal sufficient balance-a new strategy to balance baseline covariates and preserve randomness of treatment allocation. Stat Methods Med Res 2015;24:989-1002.

16 Pountos I, Georgouli T, Calori GM, et al. Do nonsteroidal antiinflammatory drugs affect bone healing? A critical analysis. ScientificWorldJournal 2012;2012:1-14.

17 Patel RA, Wilson RF, Patel PA, et al. The effect of smoking on bone healing: a systematic review. Bone Joint Res 2013;2:102-11.

18 Whelan DB, Bhandari M, Stephen D, et al. Development of the radiographic Union score for tibial fractures for the assessment of tibial fracture healing after intramedullary fixation. J Trauma 2010;68:629-32.

19 Litrenta J, Tornetta P, Mehta S, et al. Determination of radiographic healing: an assessment of consistency using rust and modified rust in Metadiaphyseal fractures. J Orthop Trauma 2015;29:516-20.

20 Hjermstad MJ, Fayers PM, Haugen DF, et al. Studies comparing numerical rating scales, verbal rating scales, and visual analogue scales for assessment of pain intensity in adults: a systematic literature review. J Pain Symptom Manage 2011;41:1073-93.

21 Laucis NC, Hays RD, Bhattacharyya T. Scoring the SF36 in orthopaedics: a brief guide. J Bone Joint Surg Am 2015;97:1628-34.

22 Lenox RH, Manji H. Lithium. In: Schatzberg AF, Nemeroff CB, eds. American psychiatric press textbook of psychopharmacology. 379. 2nd edn. Washington, DC: American Psychiatric Press, 1998.

23 Shorter E. The history of lithium therapy. Bipolar Disord 2009;11:4-9.

24 Malhi GS, Tanious M, Das P, et al. The science and practice of lithium therapy. Aust N Z J Psychiatry 2012;46:192-211.

25 Livingstone $\mathrm{C}$, Rampes $\mathrm{H}$. Lithium: a review of its metabolic adverse effects. J Psychopharmacol 2006;20:347-55.

26 Goeree ROB, Pettitt DB, Cuddy L, et al. An assessment of the burden of illness due to osteoporosis in Canada. J Soc Obstet Gynaecol Can 1996;18:15-24.

27 Cooper C, Campion G, Melton LJ. Hip fractures in the elderly: a world-wide projection. Osteoporosis Int 1992;2:285-9.

28 Vachhani K, Whyne CM, Schaffer A, et al. Perceptions of using lithium in fracture management: a survey of orthopaedic surgeons, fracture patients and the general public. BMC Musculoskelet Disord 2019;20:389. 